\title{
ECRS : Un logiciel pour l'évaluation du risque et des contre-mesures associés à l'exposition au radon dans les habitations
}

\author{
J.P. DEGRANGE ${ }^{1}$, F.P. LEVY' ${ }^{1}$, T. SCHNEIDER ${ }^{1}$
}

(Manuscrit reçu le 2 octobre 2001, accepté le 6 novembre 2001)

RÉSUMÉ ECRS (European Commission Radon Software) est un logiciel pour évaluer le risque et les contre-mesures associés à l'exposition au radon dans les habitations destiné aux micro-ordinateurs sous Windows. Il a été développé conjointement par le CEPN (Centre d'étude sur l'évaluation de la protection dans le domaine nucléaire) et le NRPB (National Radiological Protection Board) au Royaume-Uni, avec le support de la Commission européenne, DG ENV. L'objectif d'ECRS est de réaliser des calculs de risque spécifiques aux populations européennes pour différents profils d'exposition au radon, et d'évaluer, en termes de réduction de risque, l'efficacité dans les habitations de différentes contre-mesures. Ce logiciel évalue le risque de cancer du poumon associé à des expositions au radon individuelles ou collectives, selon l'approche épidémiologique ou dosimétrique. Dans les deux cas, les données démographiques spécifiques de la population sélectionnée sont utilisées et l'effet sur le risque de la consommation tabagique est pris en compte. Les principaux indicateurs de risque sont le risque de mortalité sur la vie entière et la perte d'espérance de vie. Un système de valeurs monétaires de l'année de vie perdue permet également de réaliser une analyse coût-efficacité des contre-mesures. ECRS est, en premier lieu, un outil d'évaluation permettant aux utilisateurs de se familiariser avec les risques associés au radon et les techniques de réduction de son impact sanitaire. Par ailleurs, son système de base de données, ses capacités d'analyse de sensibilité, ainsi que ses possibilitês de configuration en font un outil qui devrait permettre aux experts de l'évaluation du risque de différents pays d'Europe d'effectuer des calculs adaptés aux conditions locales d'exposition au radon.

ABSTRACT ECRS: A tool for risk assessment and evaluation of countermeasures associated with radon exposure in dwellings.

ECRS (European Commission Radon Software) is a software for risk calculation and evaluation of countermeasures associated with exposure to radon in dwellings. This Windows based tool for PCs has been jointly developped by the CEPN (Nuclear Protection Evaluation Centre) and the NRPB (National Radiological Protection Board), with support from the European Commission. The aim of ECRS is to perform lung cancer risk calculations specific to European populations for various exposure profiles and to evaluate, in terms of risk reduction, the effects of various countermeasures in dwellings. The software evaluates the risk of lung cancer associated with individual or collective exposure to radon, using either the epidemiological or the dosimetric approach. In both cases, demographic data specific to the selected population are used and the modifying effect of tobacco

\footnotetext{
'Centre d'étude sur l'évaluation de la protection dans le domaine nucléaire, BP 48, 92263 Fontenay-aux-Roses Cedex, France.
} 
consumption on the risk is adjusted for. The main risk indicators calculated are the whole life mortality excess risk and loss of life expectancy. A system of monetary value of life allows also to identify the most cost-effective countermeasure. This software is first of all an evaluation tool that allows users to gain knowledge of risks associated with radon exposure as well as the different risk reduction strategies. Finally, its sensitivity analysis capabilities and its database system should make it an easy to use tool for the risk evaluation experts of various countries to perform useful calculations, appropriate to their situations with regard to local radon patterns.

\section{Introduction}

Les effets sur la santé de l'exposition au radon des travailleurs et des membres du public sont étudiés depuis de nombreuses années. Des avancées récentes ont été réalisées dans l'évaluation du risque associé aux expositions au radon et dans la mise en ceuvre d'actions de protection dans les habitations. Néanmoins, une capacité accrue à évaluer et comprendre le risque sanitaire associé à de telles expositions peut jouer un rôle significatif dans les décisions relatives à la mise en œuvre éventuelle de contre-mesures.

Dans ce contexte, la Commission européenne a lancé un projet visant à développer un logiciel convivial s'appuyant sur les données et les modèles les plus récents en matière d'épidémiologie, de dosimétrie du radon, de démographie et d'efficacité des contre-mesures.

ECRS (European Commission Radon Software) est un logiciel pour microordinateur sous Windows. Il a été développé conjointement par le CEPN et le NRPB (National Radiological Protection Board) au Royaume-Uni, avec le support de la Commission européenne, DG ENV.

L'objectif d'ECRS est de réaliser des calculs de risque spécifiques aux populations européennes pour différents profils d'exposition au radon, et d'évaluer, en termes de réduction de risque, l'efficacité dans les habitations de différentes contre-mesures. Il contient une base de données pour un grand nombre de pays, et permet également à l'utilisateur de définir ses propres données et paramètres de modèles.

ECRS est, en premier lieu, un outil d'évaluation permettant aux utilisateurs de se familiariser avec les risques associés au radon et les techniques de réduction de son impact sanitaire. Par ailleurs, son système de base de données, ses capacités d'analyse de sensibilité, ainsi que ses possibilités de configuration en font un outil qui devrait permettre aux experts de l'évaluation du risque de différents pays 


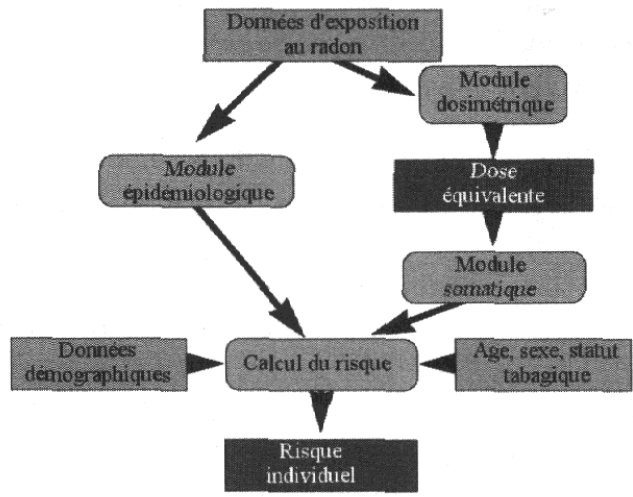

Figure 1 - Approches de calcul du risque.

Risk calculation approaches.

d'Europe d'effectuer des calculs adaptés aux conditions locales d'exposition au radon.

\section{Calculs effectués}

Le logiciel permet l'évaluation du risque de mortalité par cancer du poumon associé à des expositions au radon individuelles ou collectives.

- Le module de calcul du risque individuel détermine, pour un individu d'âge, de sexe, et de statut de consommation tabagique donnés, l'excès de risque de cancer mortel du poumon associé à un profil temporel d'exposition (passée ou future).

- Le module de calcul du risque collectif détermine l'excès de risque de cancer mortel du poumon pour chaque occupant d'une habitation, pour un profil temporel d'exposition déduit des données décrivant l'habitation, ses occupants, ainsi que les contre-mesures envisagées.

Ces calculs peuvent être effectués selon l'une ou l'autre des deux approches les plus usuelles (Fig. 1).

- L'approche épidémiologique, pour laquelle le risque de cancer mortel du poumon est directement déduit des données d'exposition au radon par l'intermédiaire d'un modèle appelé « modèle de risque épidémiologique », issu des études épidémiologiques de mineurs (notamment d'uranium).

- L'approche dosimétrique (ou somatique), pour laquelle le risque est déduit des données d'exposition au radon par l'utilisation successive d'un modèle dosimétrique et d'un modèle somatique. Le modèle dosimétrique est utilisé 
pour estimer la dose équivalente au poumon associée à une exposition donnée, alors que le modèle somatique utilise cette dose équivalente pour en déduire un risque de cancer mortel du poumon.

Dans les deux cas, l'effet de la consommation tabagique sur l'évaluation du risque de cancer du poumon associé à l'exposition au radon est pris en compte par l'utilisation de deux modèles : un modèle de risque associé à la consommation tabagique, qui ajuste le risque de cancer mortel du poumon en fonction du statut tabagique (non fumeur, fumeur, ex-fumeur) de l'individu exposé, et un modèle d'interaction tabac-radon.

Ainsi, ECRS permet d'effectuer des calculs de risque individuel à des fins de comparaison avec des estimations publiées. Il permet également d'effectuer des calculs de risque collectif qui peuvent aider, au travers d'une analyse coûtbénéfice, à sélectionner les contre-mesures les plus adaptées à une situation d'exposition donnée. Enfin, les possibilités offertes par son module de comparaison de cas de calculs facilitent l'analyse de la sensibilité des résultats aux divers paramètres de calcul.

\section{Base de données}

\subsection{Données démographiques}

La base de données d'ECRS contient des données démographiques extraites principalement de la banque de données de mortalité de l'Organisation mondiale de la santé (WHO, 1998), ainsi que des données décrivant les différents modèles utilisés dans le logiciel. Elle contient également des données de consommation tabagique de l'OMS pour les principaux États membres de l'Union européenne (WHO, 1996).

\subsection{Autres données}

Les données d'entrée et les résultats associés à un calcul spécifique sont également stockés dans la base de données et peuvent être rappelés et réexaminés à tout moment.

Les données relatives aux personnes exposées, les caractéristiques de I'habitation, la contre-mesure sélectionnée et le modèle de risque choisi sont les seules données qui doivent être introduites/sélectionnées par l'utilisateur (Fig. 2a). Tous les autres paramètres utilisés dans les calculs sont automatiquement sélectionnés par le logiciel mais des valeurs alternatives peuvent être définies et sélectionnées par l'utilisateur (Fig. 2b). 


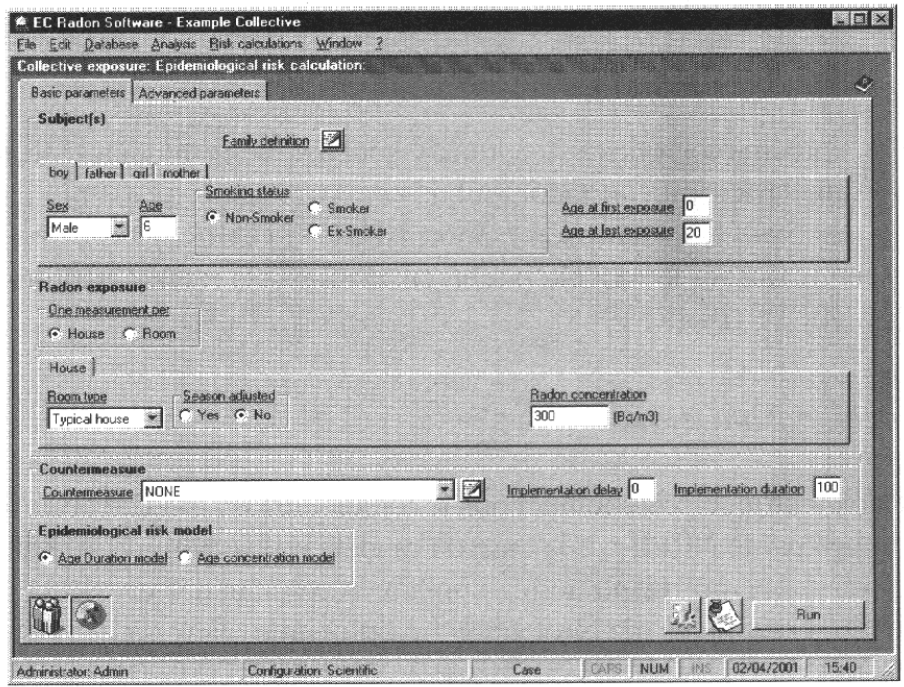

(a)

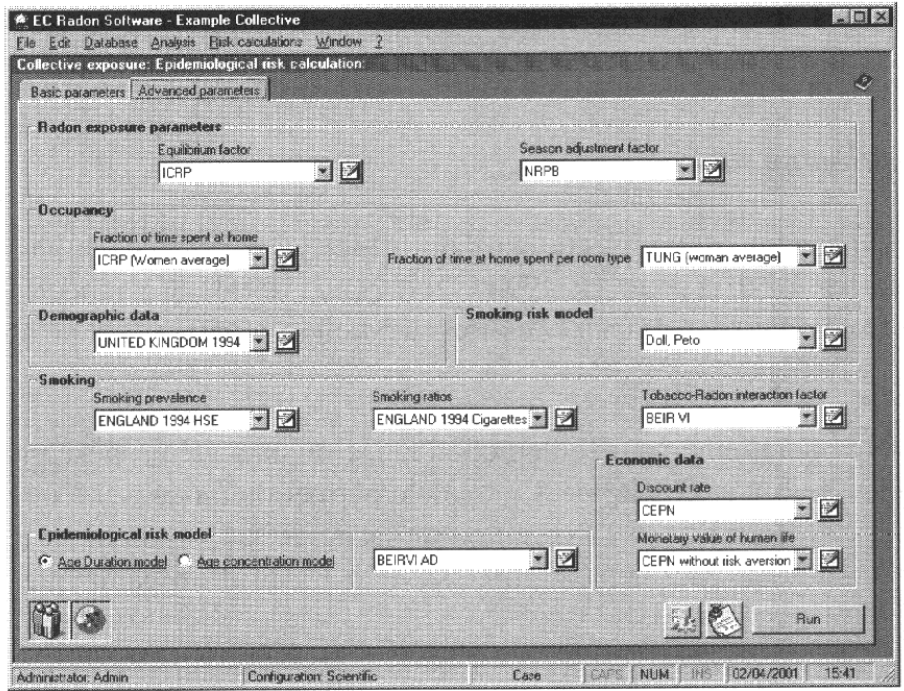

(b)

Figure 2 - Paramètres d'un calcul d'exposition collective, (a) paramètres requis, (b) paramètres avancés. Parameters for a collective exposure calculation, (a) required parameters, (b) advanced parameters. 


\section{Modèles utilisés}

\subsection{Approche épidémiologique}

Les modèles épidémiologiques sélectionnés sont les deux modèles de relation exposition-risque spécifique au radon publiés en 1999 par l'Académie nationale des sciences des États-Unis dans son rapport BEIR VI (BEIR, 1999), ainsi que, à des fins de comparaison, le modèle publié en 1988 par la même institution dans son rapport BEIR IV (BEIR, 1988).

\subsection{Approche dosimétrique}

Le modèle dosimétrique sélectionné pour le logiciel est un modèle spécifique au radon développé par le NRPB sur la base du modèle utilisé pour décrire et valider le modèle du système respiratoire humain recommandé en 1994 par la Commission internationale de protection radiologique dans sa publication 66 (ICRP, 1994).

Le modèle somatique choisi est le modèle de relation dose-effet publié en 1990 par l'Académie nationale des sciences des États-Unis dans son rapport BEIR V (BEIR, 1990).

\subsection{Exposition au tabac}

Le modèle exposition-risque sélectionné dans le logiciel pour l'exposition au tabac a été publié par Doll et Peto en 1994 (Doll et al., 1994). Ce modèle permet d'estimer l'excès de risque de cancer mortel du poumon associé au statut tabagique (fumeur et ex-fumeur).

Le modèle sélectionné pour l'interaction tabac-radon a été proposé par Lubin et al. en 1994 (Lubin et al., 1994). Ce modèle permet de modéliser l'interaction entre l'exposition au radon et l'exposition au tabac de façon purement additive, sub-multiplicative, ou purement multiplicative, à l'aide d'un paramètre unique continu.

\subsection{Exposition au radon}

L'exposition collective des occupants d'une habitation est déterminée par un modèle d'exposition utilisant des informations sur l'âge et le sexe de chacun des occupants, ainsi que sur le nombre et le type des pièces de l'habitation (cuisine, séjour et chambre). Le type de pièce est utilisé pour sélectionner les facteurs 
d'équilibre, les caractéristiques d'aérosol, ainsi que les facteurs d'occupation pertinents.

\subsection{Contre-mesures}

L'efficacité des contre-mesures sur l'exposition au radon est décrite par la réduction relative attendue de la concentration moyenne annuelle de radon dans l'habitation, ainsi que par la durée attendue de cette réduction. Des paramètres additionnels décrivent le coût d'investissement initial, le coût annuel d'utilisation et le coût de renouvellement périodique associés à la contre-mesure, afin de pouvoir calculer le coût total de la contre-mesure sur l'ensemble de la période considérée.

\section{Les résultats}

Le logiciel permet le calcul de deux types d'indicateurs du risque attribuable à l'exposition au radon :

- le risque vie entière de cancer mortel du poumon,

- la perte d'espérance de vie par cancer mortel du poumon.

Ces indicateurs sont évalués pour une exposition donnée au radon, en tenant compte, si nécessaire, de l'exposition au tabac (Fig. 3a). De plus (Fig. 3b), tous les résultats intermédiaires sont disponibles.

\subsection{Analyse coût-bénéfice}

Un système de valeurs monétaires de l'année de vie perdue est utilisé pour attribuer une valeur monétaire à la perte d'espérance de vie associée à une exposition donnée (détriment résiduel). Pour chaque situation d'exposition, le calcul de la somme du coût total (coût d'investissement et coût annuel) d'une contre-mesure potentielle et de la valeur monétaire du détriment résiduel permettent d'identifier, compte tenu des hypothèses considérées, la contre-mesure la plus coût-efficace, c'est-à-dire celle qui minimise la somme du coût de protection et du coût du détriment.

\subsection{Comparaison des résultats}

Les résultats de différents calculs peuvent être visualisés au sein d'un même tableau ou graphique afin de faciliter par exemple l'identification de la contremesure la plus efficace (Fig. 4) ou de montrer clairement l'impact sur les résultats de la variation simultanée d'un ou plusieurs paramètres. 


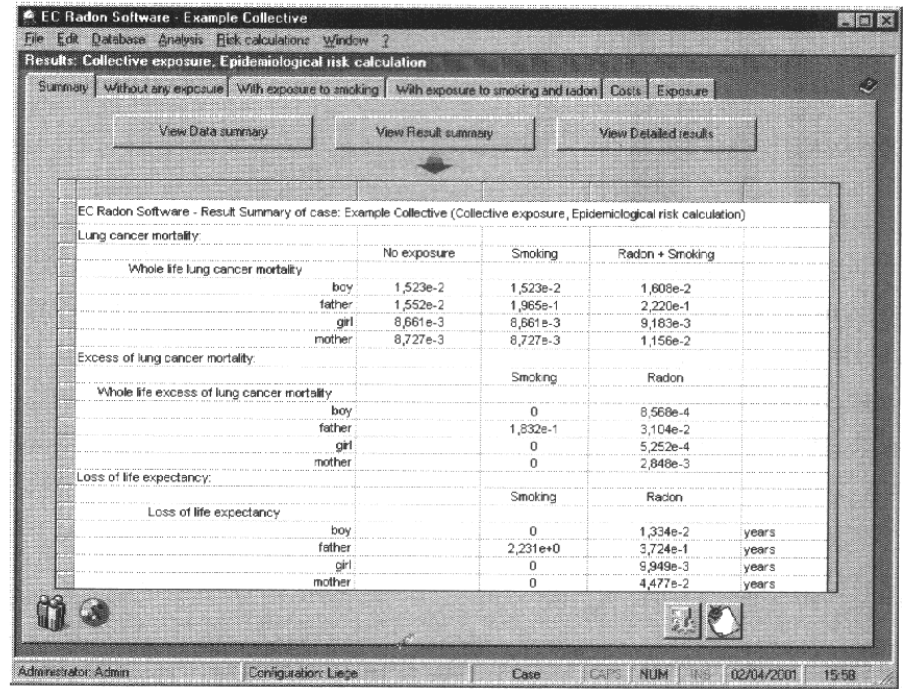

(a)

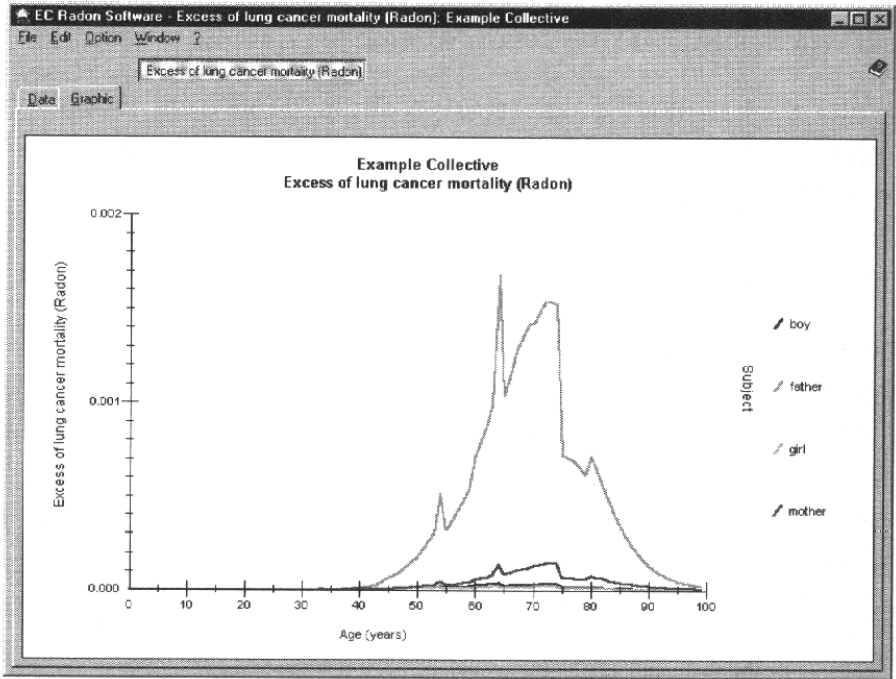

(b)

Figure 3 - Résultats d'un calcul d'exposition collective, (a) résultats résumés, (b) excès de mortalité par cancer du poumon associé à l'exposition au radon.

Results of a collective exposure calculation, (a) result summary, (b) excess of lung cancer mortality associated with radon exposure. 


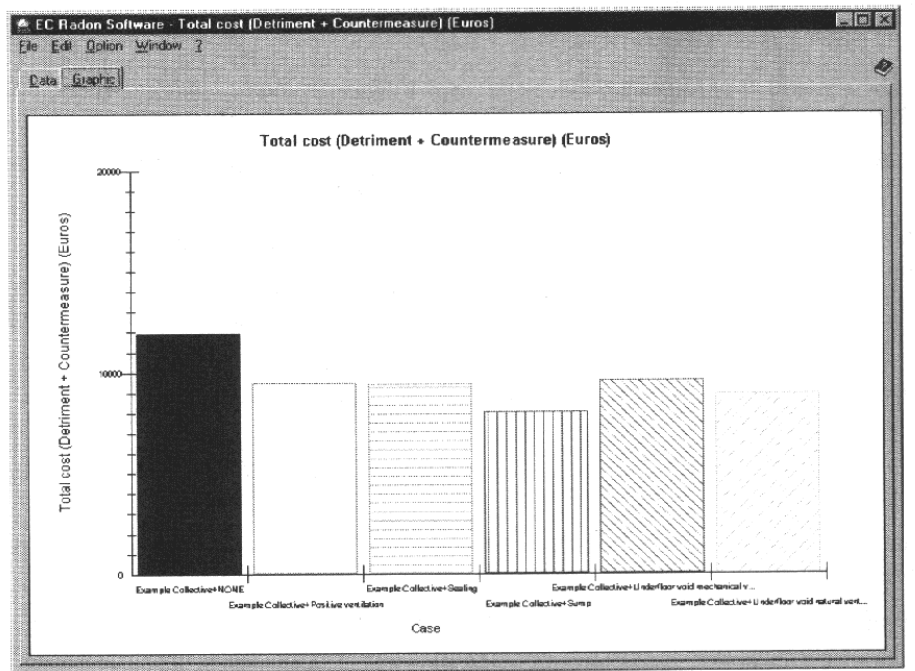

Figure 4 - Identification des contre-mesures les plus coût-efficaces.

Identification of the most cost-effective countermeasures.

\section{Autres caractéristiques}

\subsection{Documentation}

La documentation est disponible à la fois sous la forme d'un manuel et d'une aide en ligne contextuelle. Au-delà de conseils relatifs à l'utilisation du logiciel et de la description exhaustive du contenu des bases de données, le guide d'utilisateur comporte également des annexes décrivant de façon détaillée les modèles utilisés ainsi que l'ensemble des résultats (intermédiaires ou finaux) des calculs réalisés.

\subsection{Configuration matérielle et logicielle}

ECRS peut être exécuté sur tout micro-ordinateur PC à base de Pentium sous les systèmes d'exploitation suivants: Windows 95 , Windows 98 , Windows NT 4 , Windows 2000 Professionnel.

\subsection{Distribution}

Ce logiciel est distribué en version anglaise au coût de reproduction ${ }^{2}$.

\footnotetext{
${ }^{2}$ Pour plus d'information, s'adresser à Jean-Pierre Degrange, e-mail : ecrs@cepn.asso.fr
} 


\section{J.P. DEGRANGE et al.}

\section{RÉFÉRENCES}

BEIR (1988) Health effects of Radon and other internally deposited alpha-emitters, BEIR IV, National Academy Press, Washington DC, Committee on the Biological Effects of Ionizing Radiations.

BEIR (1990) Health effects of exposure to low levels of ionizing radiation, BEIR V, National Academy Press, Washington DC, Committee on the Biological Effects of Ionizing Radiations.

BEIR (1999) Health effects of exposure to Radon, BEIR VI, National Academy Press, Washington DC, Committee on Health Risks of Exposure to Radon.

Doll R. et al. (1994) Mortality in relation to smoking: 40 years observations on male British doctors, BMJ 309, 901-911.

ICRP (1994) Human respiratory tract model for radiological protection, ICRP Publication 66. Pergamon Press, Oxford.

Lubin J.H. et al. (1994) Radon and lung cancer risk: A joint analysis of 11 underground miners studies, NIH, Washington DC, Publication No. 94-3644.

WHO (1996) Tobacco alert, World Health Organisation, Geneva, special issue of the World no-tobacco day 1996.

WHO (1998) Mortality Databank, World Health Organisation, Geneva. 